\title{
Power Transfer Allocation for Open Access Using Graph Theory-Fundamentals and Applications in Systems Without Loopflow
}

\author{
Felix F. Wu, Fellow, IEEE, Yixin Ni, Senior Member, IEEE, and Ping Wei, Student Member, IEEE
}

\begin{abstract}
In this paper, graph theory is used to calculate the contributions of individual generators and loads to line flows and the real power transfer between individual generators and loads that are significant to transmission open access. Related lemmas are proved which present necessary conditions required by the method. Based on ac load flow solution a novel method is suggested which can decide downstream and upstream power flow tracing paths very fast and calculate the contribution factors of generations and loads to the line flows efficiently. The power transfer between generators and loads can also be determined. The suggested method is suitable for both active and reactive power tracings of real power systems.
\end{abstract}

Index Terms-Graph theory application, power flow tracing, transmission open access.

\section{INTRODUCTION}

$\mathbf{P}$ OWER system deregulation and transmission open access make it more and more important to calculate the contributions of individual generators and loads to line flows and the real power transfers between individual generators and loads. The results can help to allocate the total cost of transmission among all the users in an equitable way.

Methods based on dc load flow and sensitivity analysis can't consider accurately the reactive power transfer allocation and system nonlinearity. In [1], [2] a novel method is suggested to solve the problem however a matrix inverse calculation is required which is time consuming especially for a large scale power system. In [3] another approach is presented which introduces some new concepts such as domain, common, link and state graph and is suitable for large-scale power system applications. However there are no clear declaration and proof of the conditions required for the method.

In this paper graph theory [4], [5] is suggested to solve the problem. Graph theory is quite mature and especially suitable to tackle such network topology related issue. In our case directed graph is used. The vertices of the graph are system buses and the edges of the graph are lines and transformers. The direction of each edge is the direction of power flow inside. The directed graph of active power flow may be different from that of reactive power flow in edge directions.

Related lemmas are presented and proved first which presents the necessary conditions to guarantee the feasibility of the sug-

Manuscript received February 19, 1999; revised June 28, 1999.

The authors are with the Department of Electrical and Electronic Engineering, The University of Hong Kong, Hong Kong SAR.

Publisher Item Identifier S 0885-8950(00)07723-3. gested method in power flow tracing. Then based on the widely used bus-line incident matrix, downstream and upstream tracing sequences are determined at ease. A novel approach is suggested to calculate the contribution factors of individual generations and loads to the line flows respectively. The real power transfer between individual generators and loads can also be determined. The method can be used for both active and reactive power transfer allocations. Based on ac load flow solution it can consider system nonlinearity accurately. The suggested method is very efficient and suitable for use in real power systems.

The paper is organized as follows. In Section II the basic assumptions are described. Related lemmas are presented and proved. Directed graphs for upstream and downstream tracings, the corresponding bus-inflow-line and bus-outflow-line incident matrices and their usage in power flow tracing are introduced. In Section III through upstream and downstream tracings, contribution factors of individual generators and loads to line flows are calculated respectively. The power transfers between generators and loads are determined as well. A simple example is used in Sections II and III to help the illustration of the method. Section IV presents the test results from the IEEE 30-bus system. Conclusions are made in Section V.

\section{Fundamentals OF LOAD Flow TRACiNG}

In order to simplify the problem, we first make the following basic assumptions:

- An ac load flow solution is available from on-line state estimation or off-line system analysis. The studied system has finite number of buses. It is operated properly and there is no loop flow in the system.

- Real power and reactive power required by transmission line resistance, reactance and charging capacitance have been moved to the line terminal buses (see Section III for details) and modeled as "equivalent loads" according to ac load flow solution. Therefore the line active and reactive power flows keep constant along the line, each edge has a definite direction and the network is "lossless."

- A generator has the priority to provide power to the load on the same bus. The remaining power will enter the network to supply other loads in the network to avoid unnecessary losses. It is true even according to a transaction contract a generator does not sell electricity to the local load. This is because electricity has no label and system operators have the authority to dispatch the power flow. Therefore the buses of a network can be classified as generator buses, 


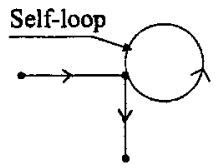

(a) Self-loop

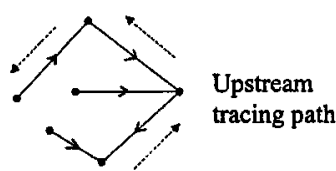

(b) Upstream tracing path

Fig. 1. Diagram for proof of lemma I (a) Self-loop (b) Upstream tracing path.

load buses and network buses based on their net injection to the system. This concept can be applied to both active and reactive power flows.

- The flows of electricity obey the proportional-sharing rule [1]-[3].

Based on the assumptions made above we can prove the following two lemmas:

Lemma 1: A lossless, finite-nodes power system without loop flow has at least one pure source, i.e. a generator bus with all incident lines carrying outflows.

This lemma will be proved below. It guarantees to start and continue a downstream tracing from an existing pure source.

Lemma 2: A lossless, finite-nodes power system without loop flow has at least one pure sink, i.e. a load bus with all incident lines carrying inflows.

This lemma can be proved similarly as lemma 1 . It guarantees to start and continue an upstream tracing from an existing pure sink.

Proof of Lemma 1: We start from any bus with an incident line carrying inflows. Along this line we go upstream and come to a "next" bus. Here we assume in power system there is no self-loop [see Fig. 1(a)]. If there is no incident line carrying inflows to the next bus, then it is an existing pure source already. If there is at least one incident line carrying inflow to the next bus, then we can continue to go upstream along the line to another bus [see Fig. 1(b)]. This upstream tracing can continue if there is no pure source confronted. If the upstream bus is always a new bus, this will lead to the conclusion that the system has infinite buses. If the upstream bus is an old bus, that is a bus appeared in the previous tracing path, then a loop flow is existing in the system which conflicts with the assumption. Therefore a pure source does exist to end the upstream tracing.

Proof of Lemma 2: We start from any bus with an incident line carrying outflows. Along this line we go downstream and come to a "next" bus (still we assume there is no self-loop). If there is no incident line to carry outflows from the next bus, then it is an existing pure sink already. If there is at least one incident line carrying outflow from the next bus, then we can continue to go downstream along the line to another bus. This downstream tracing can continue if there is no pure sink confronted. If the downstream bus is always a new bus, this will lead to the conclusion that the system has infinite buses. If the downstream bus is an old bus, that is a bus appeared in the previous tracing path, then a loop flow is existing in the system which conflicts with the assumption. Therefore a pure sink does exist to end the downstream tracing.

Based on the two lemmas, we can use bus-line incident matrix to form bus-inflow-line and bus-outflow-line incident matrices respectively and determine the pure sink and pure source of the

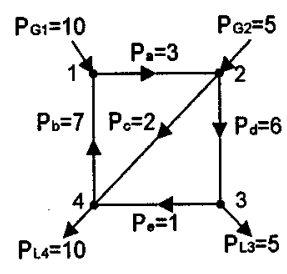

(a) System graph (P: MW)

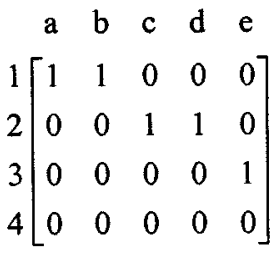

(c) System BOLIM

a
$2\left[\begin{array}{ccccc}1 & 1 & 0 & 0 & 0 \\ -1 & 0 & 1 & 1 & 0 \\ 0 & 0 & 0 & -1 & 1 \\ 0 & -1 & -1 & 0 & -1\end{array}\right]$

(b) System BLIM

a
$\frac{b}{2}\left[\begin{array}{lllll}0 & 0 & 0 & 0 & 0 \\ 1 & 0 & 0 & 0 & 0 \\ 0 & 0 & 0 & 1 & 0 \\ 0 & 1 & 1 & 0 & 1\end{array}\right]$

(d) System BILIM
Fig. 2. Sample system diagram and matrices (a) System graph (P: MW) (b) System BLIM (c) System BOLIM (d) System BILIM.

studied system quickly. This can be shown through an example [see Fig. 2(a)]. The system has 4 buses (buses 1 to 4 ) and 5 lines (lines a to e). The active power flow is used to form corresponding directed graph. The bus-line incident matrix (BLIM) is shown in Fig. 2(b).

BLIM can split into bus-outflow-line and bus-inflow-line incident matrices easily. The former is formed by all elements " -1 " in BLIM. The latter is formed by all elements " 1 " in BLIM and then all elements " -1 " are changed to " 1 ." The resultant two matrices are shown in Fig. 2 where Fig. 2(c) is the bus-outflow-line incident matrix (BOLIM) and Fig. 2(d) is the bus-inflow-line incident matrix (BILIM).

The row with full zeros in BOLIM corresponds to pure sink bus since there are no outflows from the bus. The row with full zeros in BILIM corresponds to pure source bus since there are no inflows to the bus. It is clear that bus 4 is a pure sink and bus 1 is a pure source in our example. A system might have more than one pure sink and pure source.

The upstream and downstream tracing sequence is determined based on the two matrices as follows.

Downstream tracing sequence:

We start from a pure source (bus 1 for our case). Its outflows will bring generator power forward to their downstream buses. Delete the source bus and its incident lines (This is done by delete the corresponding pure source row 1 and corresponding columns of its incident lines, i.e. columns with element 1 in the row 1 of BOLIM). We have a new graph that is a sub-graph of the original one. Then find the new pure source bus in the current graph and its incident (outflow) lines (Since the current graph still satisfies the conditions of lemma 1, a new pure source bus must be existed.) and repeat the deleting process described above again. This process will continue till there is no line existing in the current graph. Then all the remaining buses are pure sinks. The inter-mediate BOLIM and BILIM in downstream tracing for our example are shown in Fig. 3.

Upstream tracing sequence:

Upstream tracing can be performed similarly through starting from a pure sink. The tracing ends when there is no 


\begin{tabular}{|c|c|c|c|c|c|c|c|c|c|}
\hline \multicolumn{4}{|c|}{ BOLIM } & \multicolumn{6}{|c|}{ BILIM } \\
\hline a & $\mathrm{b}$ & c d & $\mathrm{e}$ & & $\mathrm{a}$ & $b$ & c & $\mathrm{d}$ & e \\
\hline $1\lceil 1$ & 1 & $\begin{array}{ll}0 & 0\end{array}$ & $0]$ & & 0 & 0 & 0 & 0 & 07 \\
\hline 0 & 0 & 11 & 0 & 2 & 1 & 0 & 0 & 0 & 0 \\
\hline 0 & 0 & 00 & 1 & 3 & 0 & 0 & 0 & 1 & 0 \\
\hline $4[0$ & 0 & $\begin{array}{ll}0 & 0\end{array}$ & 0 & & 0 & 1 & 1 & 0 & $1]$ \\
\hline
\end{tabular}

Step 1: Pure source is bus 1 . Lines $a$ and $b$ are deleted.

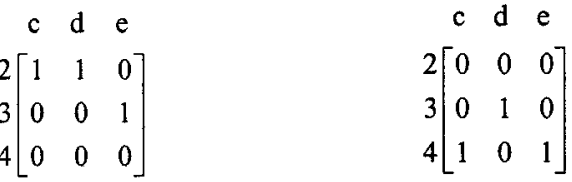

Step 2: Pure source is bus 2. Lines $\mathrm{c}$ and $\mathrm{d}$ are deleted.

$\begin{array}{cc}e & e \\ 4\left[\begin{array}{l}1 \\ 0\end{array}\right] & 3\left[\begin{array}{l}0 \\ 1\end{array}\right]\end{array}$

Step 3: Pure source is bus 3. Line e is deleted.

Fig. 3. Downstream tracing sequence Step 1: Pure source is bus 1. Lines a and $\mathrm{b}$ are deleted. Step 2: Pure source is bus 2. Lines c and d are deleted. Step 3 : Pure source is bus 3 . Line 3 is deleted.

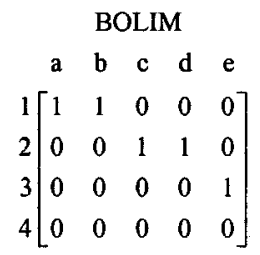

\begin{tabular}{|c|c|}
\hline \multicolumn{2}{|c|}{ BILIM } \\
\hline b c & d \\
\hline $\begin{array}{ll}0 & 0\end{array}$ & 0 \\
\hline $\begin{array}{ll}0 & 0\end{array}$ & 0 \\
\hline 00 & 1 \\
\hline & 0 \\
\hline
\end{tabular}

Step 1: Pure sink is bus 4 . Lines $b, c$ and e are deleted.

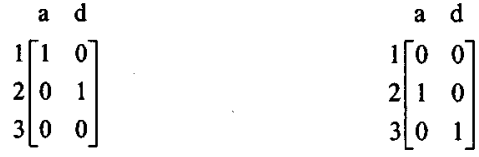

Step 2: Pure sink is bus 3. Line $d$ is deleted.

$\begin{array}{cc}a & a \\ 2\left[\begin{array}{l}1 \\ 0\end{array}\right] & 1\left[\begin{array}{l}0 \\ 1\end{array}\right]\end{array}$

Step 3: Pure sink is bus 2. Line a is deleted.

Fig. 4. Upstream tracing sequence Step 1: Pure sink is bus 4. Lines b, c and e are deleted. Step 2: Pure sink is bus 3: Line $d$ is deleted. Step 3: Pure sink is bus 2. Line a is deleted.

lines existing in the current graph. Then all the remaining buses are pure sources. The inter-mediate BOLIM and BILIM in upstream tracing for our example are shown in Fig. 4.

\section{CONTRIBUtion AND EXTRACTION FACTORS CALCULATIONS}

\section{A. Downstream Tracing}

The downstream tracing (DSTR) is used for calculating the contribution factors of individual generators to line flows and loads. The tracing path is determined by the method suggested in Section II and starting from a pure source. For our example, the DSTR sequence is bus $1 \rightarrow$ bus $2 \rightarrow$ bus $3 \rightarrow$ bus 4 . The state variable in DSTR is the net generator power.
To solve the problem we first build up two matrices. One is extraction factor matrix of lines and loads from bus total passing power. The other is contribution factor matrix of generators to bus total passing power. The product of these two matrices constitutes the contribution factors of generators to line flows and loads.

Extraction factors of lines from bus total passing power

We first build up an extraction factor matrix $\left(A_{l}\right)$ of lines from total passing power of their upstream buses, i.e. $P_{l}=A_{l} \cdot P$. Here $P_{l}$ is the vector of line power ( $P_{a}$ through $P_{e}$ for our case); $P$ is the vector of bus total passing power in the bus sequence of downstream tracing and calculated from ac load flow solution. The nonzero element in $A_{l}$ is calculated as follows:

$$
\left(A_{l}\right)_{\text {line } j, \text { bus } i}=\frac{\text { line } j \text { 's power flow }}{\text { bus i's total passing power } P_{i}}
$$

where bus $i$ is the upstream bus of line $j . P_{i}$ includes both line inflow power and net generator injection power to bus $i$ calculated from load flow solution. For our case [see Fig. 2(a)] $P=(108610)^{\mathrm{T}}$ and $A_{l}$ is

$$
A_{l}=\begin{gathered}
a \\
b \\
c \\
d \\
e
\end{gathered}\left[\begin{array}{cccc}
3 / 10 & P_{2} & P_{3} & P_{4} \\
7 / 10 & 0 & 0 & 0 \\
0 & 2 / 8 & 0 & 0 \\
0 & 6 / 8 & 0 & 0 \\
0 & 0 & 1 / 6 & 0
\end{array}\right]
$$

Similarly we can form an extraction factor matrix $\left(A_{L}\right)$ of loads from total passing power of their located buses, i.e. $P_{L}=A_{L} \cdot P$. The vector of load power $P_{L}$ takes the same sequence as vector $P$, therefore matrix $A_{L}$ will be a diagonal matrix where

$$
A_{L_{i i}}= \begin{cases}{ }^{\text {net load power on bus } i} & i \notin \text { net load buses } \\ P_{i} & i \notin \text { net load buses }\end{cases}
$$

For our example $A_{L}$ will be

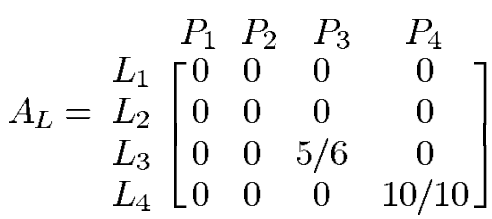

If we merge matrices $A_{l}$ and $A_{L}$ together as extraction factor matrix of lines and loads from bus total passing power, we have

$$
A=\left[\begin{array}{l}
A_{l} \\
A_{L}
\end{array}\right]
$$

we can prove that each element in $A$ is nonnegative; the sum of elements in each column of matrix $A$ equals one; there is one and only one nonzero element in each row of sub-matrix $A_{l}$; and the nonzero elements in sub-matrix $A_{l}$, are corresponding to the nonzero elements in BOLIM.

Contribution factors of generators to bus total passing power 
We define contribution factor matrix $(B)$ of generators to bus total passing power as

$$
P=B \bullet P_{G}
$$

where the vector of generator power $P_{G}\left(P_{G i}=0\right.$ if bus $i$ is not a net generator bus) and the vector of bus total passing power $P$ have same bus sequence as that in downstream tracing. Matrix $B$ is formed row by row. It is clear from DSTR that for a certain bus $i$ only its upstream generators have impacts on its total injection (passing) power, so the upper triangular elements of matrix $B$ corresponding to the downstream generator effects on upstream bus total injection power should be zero. The diagonal elements $B_{\text {bus } i, b u s i}$ will be 1 (or 0 ) if bus $i$ is (or is not) a net generator bus. A nongenerator bus $k$ will not have any contribution to the total injection (passing) power of any buses. The elements in matrix $B$ can be calculated by equation (1):

$$
\begin{aligned}
& \text { Bbus-i,bus-k} \begin{array}{ll}
1 & (k=i, k \in \text { net gen. buses }) \\
0 & (k=i, k \notin \text { net gen. buses }) \\
0 & (k>i) \\
0 & (k<i, k \notin \text { net gen. buses }) \\
\sum_{l_{j} \varepsilon i}\left(A_{l_{j}}-m\right. & \\
\left.\cdot B_{m-k}\right) & (k<i, k \in \text { net gen. buses })
\end{array}
\end{aligned}
$$

where " $k<i$ " means $k$ is an upstream bus of bus $i$, and " $k>i$ " means $k$ is a downstream bus of bus $i$. The last expression is for the lower triangular nonzero elements. The term " $l_{j} \varepsilon i$ " means line $j$ is an inflow line of bus $i . A_{l_{j}-m}$ is the unique nonzero element corresponding to line $j$ in matrix $A_{l}$ with bus $m$ as its upstream terminal. $B_{m-k}$ is the element in matrix $B$ already calculated which represents the contribution of generator $k$ to the total injection power of bus $m$. The product $\left(A_{l_{j}-m} \bullet B_{m-k}\right)$ represents the contribution of generator $k$ to the total injection power of bus $i$ through line $j$ (from bus $m$ to bus $i$ ). For our example it is easy to form matrix $B$ row by row according to equation (1). The resultant $B$ is shown at the bottom of the page.

Now we are ready to determine the contribution factors of individual generators to line flows and loads. Based on matrices $A_{l}, A_{L}$ and $B$, since $P_{l}=A_{l} P$ and $P=B P_{G}$, so we have

$$
P_{l}=A_{l} P=A_{l} B P_{G}=K_{l G} P_{G}
$$

where $K_{l G}$ is the contribution factor matrix of generators to line flows. Similarly since $P_{L}=A_{L} P$ and $P=B P_{G}$, so we have

$$
P_{L}=A_{L} P=A_{L} B P_{G}=K_{L G} P_{G}
$$

where $K_{L G}$ is the contribution factor matrix of generators to loads which shows that each load gets their real power supply from which generators and at what contribution factors. For our example we have

$$
\begin{aligned}
& P_{G 1} \quad P_{G 2} \quad P_{G 3} \quad P_{G 4} \\
& \left.K_{l G}=A_{l} B=\begin{array}{c}
a \\
b \\
c
\end{array} \begin{array}{ccccc}
3 / 10 & 0 & 0 & 0 \\
7 / 10 & 0 & 0 & 0 \\
d & d / 40 & 1 / 4 & 0 & 0 \\
9 / 40 & 3 / 4 & 0 & 0 \\
3 / 80 & 1 / 8 & 0 & 0
\end{array}\right]
\end{aligned}
$$

and

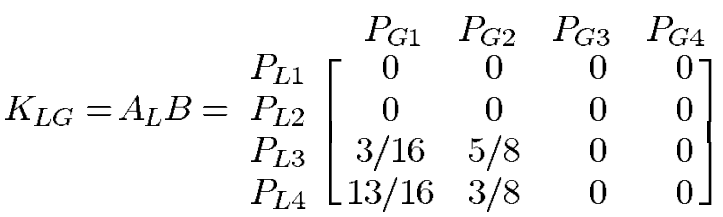

However we are not able to get $P_{G}=K_{G L} P_{L}$ and $P_{l}=$ $K_{I L} P_{L}$ from the downstream tracing since the state variable in DSTR is the generator power. These relations can only be obtained from the upstream tracing which takes the load power as the state variable.

\section{B. Upstream Tracing}

The upstream tracing (USTR) is used for calculating the extraction factors of loads from line flows and generators. The tracing path is determined by the method suggested in Section II and beginning from a pure sink bus. For our example, the USTR sequence is bus $4 \rightarrow$ bus $3 \rightarrow$ bus $2 \rightarrow$ bus 1 . Usually USTR can takes the reverse sequence of DSTR.

Similarly we first build up two matrices. One is contribution factor matrix of lines and generators to bus total passing power. The other is extraction factor matrix of loads from bus total passing power. The product of these two matrices constitutes the extraction factors of loads from line flows and generators.

Contribution factors of lines to bus total passing power

We first build up a contribution factor matrix $\left(C_{l}\right)$ of lines to the total passing power of their downstream terminal buses. i.e.

$$
P_{i}=C_{l} \bullet P
$$

$$
B=\begin{array}{cccc}
P_{1} \\
P_{2} \\
P_{3} \\
P_{4}
\end{array}\left[\begin{array}{cccc}
P_{G 1} & P_{G 2} & P_{G 3} & P_{G 4} \\
1 & 0 & 0 & 0 \\
\frac{3}{10} \times 1=\frac{3}{10} & 1 & 0 & 0 \\
\frac{6}{8} \times \frac{3}{10}=\frac{9}{40} & \frac{6}{8} \times 1=\frac{6}{8} & 0 & 0 \\
\frac{7}{10} \times 1+\frac{2}{8} \times \frac{3}{10}+\frac{1}{6} \times \frac{9}{40}=\frac{3}{16} & \frac{2}{8} \times 1+\frac{1}{6} \times \frac{6}{8}=\frac{3}{8} & 0 & 0
\end{array}\right]
$$


Here $P_{l}$ and $P$ has the same definition as DSTR but the vector $P$ of total passing power of buses is in the bus sequence of USTR. The nonzero element in $C_{l}$ is determined as follows:

$$
\left(C_{l}\right)_{\text {line } j, \text { bus } i}=\frac{\text { line } j \text { 's power flow }}{\text { bus i's total passing power } P_{i}}
$$

where bus $i$ is the downstream terminal bus of line $j$. For our case $C_{l}$ is [see Fig. 2(a)]:

$$
C_{l}=\begin{gathered}
a \\
b \\
c \\
d \\
d \\
e
\end{gathered}\left[\begin{array}{cccc}
P_{4} & P_{3} & P_{2} & P_{1} \\
7 / 10 & 0 & 3 / 8 & 0 \\
2 / 10 & 0 & 0 & 0 \\
0 & 6 / 6 & 0 & 0 \\
1 / 10 & 0 & 0 & 0
\end{array}\right]
$$

Similarly we can form a contribution factor matrix $\left(C_{G}\right)$ of generators to the bus total passing power, i.e. $P_{G}=C_{G} \bullet P$. If the vector of generator power $P_{G}$ uses the same USTR sequence as vector $P$, matrix $C_{G}$ will be a diagonal matrix and

$$
C_{G_{i i}}= \begin{cases}0 & i \notin \text { net gen. buses } \\ \frac{\text { net gen. power on bus } i}{P_{i}} & i \in \text { net gen. buses }\end{cases}
$$

For our example $C_{G}$ will be

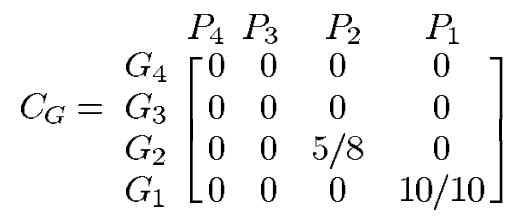

If we merge matrices $C_{l}$ and $C_{G}$ together as contribution matrix $C$ of lines and generators to bus passing power, we have

$$
C=\left[\begin{array}{c}
C_{l} \\
C_{G}
\end{array}\right]
$$

It is easy to prove the following. Each element in $C$ is nonnegative. The sum of elements in each column of matrix $C$ equals one. There is one and only one nonzero element in each row of sub-matrix $C_{l}$. And the nonzero elements in sub-matrix $C_{l}$ are corresponding to the nonzero elements in BILIM.

Extraction factors of loads from bus total passing power

We define extraction factor matrix $(D)$ of loads from bus total passing power as

$$
P=D \bullet P_{L}
$$

where the vector of load power $P_{L}\left(P_{L i}=0\right.$ if bus $i$ is not a net load bus) and the vector of bus total passing power $P$ have same bus sequence as that in USTR. Matrix $D$ will be formed row by row. It is clear that for a certain bus $i$ only its downstream loads have impacts on its total outflow (passing) power, so the upper triangular elements of matrix $D$ corresponding to the upstream load effects on downstream bus total outflow (passing) power should be zero. The diagonal element $D_{b u s i}$, bus $i$ will be 1 (or 0 ) if bus $i$ is (or is not) a net load bus. A nonload bus $k$ will not have any contribution to the total outflow power of any buses. The elements in matrix $D$ can be calculated by equation (4):

$$
\begin{array}{ll}
D_{b u s^{-i}, \text { bus }-k} & (k=i, k \in \text { net load. buses }) \\
0 & (k=i, k \notin \text { net load. buses }) \\
0 & (k>i) \\
0 & (k<i, k \notin \text { net load buses }) \\
\sum_{l_{j \varepsilon i}}(4) &
\end{array}
$$

where " $k<i$ " means bus $k$ is a downstream bus of bus $i$ and " $k>i$ " means bus $k$ is an upstream bus of bus $i$. The last expression in equation (4) is for the lower triangular nonzero elements. The term " $l_{j \varepsilon i}$ " means that line $j$ is an outflow line of bus $i . C_{l_{j}-m}$ is the unique nonzero element corresponding to line $j$ (from bus $i$ to bus $m$ ) in matrix $C_{l}$. $D_{m-k}$ is the element in matrix $D$ already calculated which represents the extraction factor of load $k$ from the total passing power of bus $m$. The product $\left(C_{l_{j}-m} \bullet D_{m-k}\right)$ represents the extraction of load $k$ from the total outflow power of bus $i$ through line $j$ (from bus $i$ to bus $m$ ). For our example it is easy to form matrix $D$ row by row. According to equation (4) the resultant $D$ is

$$
\begin{aligned}
& D=\left[\begin{array}{cccc}
1 & 0 & 0 & 0 \\
\frac{1}{10} \times 1=\frac{1}{10} & 1 & 0 & 0 \\
\frac{2}{10} \times 1+\frac{6}{6} \times \frac{1}{10}=\frac{3}{10} & \frac{6}{6} \times 1=1 & 0 & 0 \\
\frac{7}{10} \times 1+\frac{3}{8} \times \frac{3}{10} & \frac{3}{8} \times 1 & 0 & 0
\end{array}\right] \\
& \begin{array}{llll}
P_{L 4} & P_{L 3} & P_{L 2} & P_{L 1}
\end{array} \\
& =\begin{array}{r}
P_{4} \\
P_{3} \\
P_{2} \\
P_{1}
\end{array}\left[\begin{array}{cccc}
1 & 0 & 0 & 0 \\
1 / 10 & 1 & 0 & 0 \\
3 / 10 & 1 & 0 & 0 \\
13 / 16 & 3 / 8 & 0 & 0
\end{array}\right]
\end{aligned}
$$

Now we are ready to determine the extraction factors of individual loads from line flows and generators. Based on matrices $C_{l}, C_{L}$ and $D$, since $P_{l}=C_{l} P$ and $P=D P_{L}$, so we have

$$
P_{l}=C_{l} P=C_{l} D P_{L}=K_{l L} P_{L}
$$

where $K_{l L}$ is the extraction factor matrix of loads from line flows. Similarly since $P_{G}=C_{G} P$ and $P=D P_{L}$, so we have

$$
P_{G}=C_{G} P=C_{G} D P_{L}=K_{G L} P_{L}
$$

where $K_{G L}$ is the extraction factor matrix of loads from generators which shows how each generators provides power supply to individual loads. For our example we have

$$
K_{l L}=C_{l} D=\begin{gathered}
P_{L 4} \\
a \\
b \\
c \\
c \\
d \\
d \\
e
\end{gathered}\left[\begin{array}{cccc}
9 / 80 & 3 / 8 & 0 & 0 \\
7 / 10 & 0 & 0 & 0 \\
2 / 10 & 0 & 0 & 0 \\
1 / 10 & 1 & 0 & 0 \\
1 / 10 & 0 & 0 & 0
\end{array}\right]
$$

and

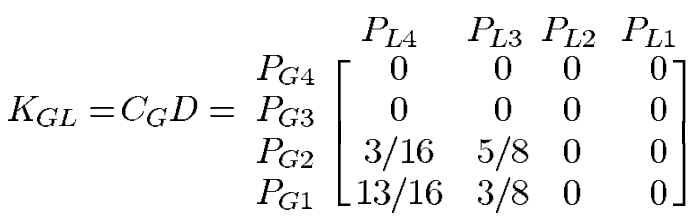


Substituting $P_{L}$ in equation (6), or $P_{G}$ in equation (3), we have the real power transfer between generators and loads as follows (in MW):

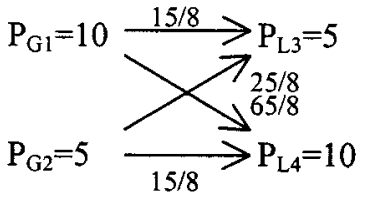

It should be noticed that the sum of elements in each column of matrices $K_{G L}$ and $K_{L G}$ equals 1 which means there is a nonindependent equation in matrix equations (3) and (6).

\section{Handling Power Required by Line $R, X$ and $C$}

A line can be considered as its " $\pi$ "-equivalence and its charging capacitance effects can be included in its terminal bus loads according to load flow solution. Line resistance (or reactance) can be treated similarly as follows. If active (or reactive) power flow at the two terminals of the line are in the same direction, the line active (or reactive) power loss can be moved to the sending bus and the line flow will equal to receiving end power flow. The line active (or reactive) power loss can also be moved to the receiving bus, then the line flow will equal to the sending end power flow. However if the two terminals of the line have different active (or reactive) power flow directions, i.e. both terminals inject power to the line, the two injection powers should be treated as equivalent loads on the sending and receiving buses respectively. The resultant line flow will be zero and treated as open circuit. This process is to guarantee that the power flow along the line keeps constant and the line flow has a definite direction.

If loop flows exist in a real power system, the upstream or downstream tracing can detect their existence (i.e. when there is no row with full zero elements in current BOLIM or there is no row with full zero elements in current BILIM).

However to determine the loop flow path and to evaluate the amount of the loop flow are not easy, especially when the loop flows have complicated paths. The issue needs further investigation.

\section{TEST ON IEEE 30-Bus SysteM}

In order to test the feasibility and the accuracy of the algorithm proposed above in real world system, computer software has been developed and tested on the IEEE 30-bus system. The active power flow tracing results are shown in Table I, with the system load flow results attached in Appendix.

In Table I, loads on various buses are listed. The power supplies from individual generators to each load are calculated through the suggested power tracing method. Both upstream and downstream tracings yield the same results which are presented in Table I as well. Table I also shows the loss sharing by individual generators and the balance of the system power. The new method is proved to be very simple and quite efficient in power flow tracing.
TABLE I

ANALYSIS OF TRANSFERRED POWER (MW)

\begin{tabular}{|c|c|c|c|c|c|c|c|}
\hline Bus & Load & $\begin{array}{c}\text { Supplied } \\
\text { by } \\
\text { Gen. } 1\end{array}$ & $\begin{array}{c}\text { Supplied } \\
\text { by } \\
\text { Gen. } 2\end{array}$ & $\begin{array}{c}\text { Supplied } \\
\text { by } \\
\text { Gen. } 5\end{array}$ & $\begin{array}{c}\text { Supplied } \\
\text { by } \\
\text { Gen. } 8\end{array}$ & $\begin{array}{c}\text { Supplied } \\
\text { by } \\
\text { Gen. } 11\end{array}$ & $\begin{array}{l}\text { Supplied } \\
\text { by } \\
\text { Gen. } 13\end{array}$ \\
\hline 1 & 0.00 & 0.00 & 0.00 & 0.00 & \begin{tabular}{|l|}
0.00 \\
\end{tabular} & 0.00 & 0.00 \\
\hline 2 & 21.70 & 0.00 & 21.70 & 0.00 & 0.00 & 0.00 & 0.00 \\
\hline 3 & 2.40 & 2.40 & 0.00 & 0.00 & 0.00 & 0.00 & $\overline{0.00}$ \\
\hline 4 & 7.60 & 6.74 & 0.86 & 0.00 & 0.00 & 0.00 & $\overline{0.00}$ \\
\hline 5 & 94.20 & 50.77 & 18.77 & 24.56 & 0.10 & 0.00 & $\overline{0.00}$ \\
\hline 6 & 0.00 & 0.00 & 0.00 & 0.00 & 0.00 & 0.00 & 0.00 \\
\hline 7 & 22.80 & 18.15 & 4.46 & 0.00 & 0.19 & 0.00 & 0.00 \\
\hline 8 & 30.00 & 0.00 & 0.00 & 0.00 & 30.00 & 0.00 & 0.00 \\
\hline 9 & 0.00 & 0.00 & 0.00 & 0.00 & 0.00 & 0.00 & 0.00 \\
\hline 10 & 5.80 & 2.78 & 0.68 & 0.00 & 0.03 & 2.31 & 0.00 \\
\hline 11 & 0.00 & 0.00 & 0.00 & 0.00 & 0.00 & 0.00 & 0.00 \\
\hline 12 & 11.20 & 6.02 & 0.76 & 0.00 & 0.00 & 0.00 & $\overline{4.42}$ \\
\hline 13 & 0.00 & 0.00 & 0.00 & 0.00 & 0.00 & 0.00 & 0.00 \\
\hline 14 & 6.20 & 3.33 & 0.42 & 0.00 & 0.00 & 0.00 & 2.45 \\
\hline 15 & 8.20 & 4.41 & 0.56 & 0.00 & 0.00 & 0.00 & 3.23 \\
\hline 16 & 3.50 & 1.88 & 0.24 & 0.00 & 0.00 & 0.00 & 1.38 \\
\hline 17 & 9.00 & 4.49 & 0.91 & 0.00 & 0.03 & 2.34 & 1.23 \\
\hline 18 & 3.20 & 1.72 & 0.22 & 0.00 & 0.00 & 0.00 & 1.26 \\
\hline 19 & 9.50 & 4.69 & 1.00 & 0.00 & 0.04 & 2.84 & $\overline{0.93}$ \\
\hline 20 & 2.20 & 1.05 & 0.26 & 0.00 & 0.01 & 0.88 & $\overline{0.00}$ \\
\hline 21 & 17.50 & 8.39 & 2.05 & 0.00 & 0.09 & 6.97 & $\overline{0.00}$ \\
\hline 22 & 0.00 & 0.00 & 0.00 & 0.00 & 0.00 & 0.00 & 0.00 \\
\hline 23 & 3.20 & 1.72 & 0.22 & 0.00 & 0.00 & 0.00 & 1.26 \\
\hline 24 & 8.70 & 4.39 & 0.98 & 0.00 & 0.29 & 2.45 & 0.59 \\
\hline 25 & 0.00 & 0.00 & 0.00 & 0.00 & 0.00 & 0.00 & 0.00 \\
\hline 26 & 3.50 & 2.12 & 0.52 & 0.00 & 0.86 & 0.00 & 0.00 \\
\hline 27 & 0.00 & 0.00 & 0.00 & 0.00 & 0.00 & 0.00 & 0.00 \\
\hline 28 & 0.00 & 0.00 & 0.00 & 0.00 & 0.00 & 0.00 & 0.00 \\
\hline 29 & 2.40 & 1.45 & 0.36 & 0.00 & 0.59 & 0.00 & 0.00 \\
\hline 30 & 10.60 & 6.41 & 1.57 & 0.00 & 2.62 & 0.00 & 0.00 \\
\hline Loss & 7.19 & 5.72 & 1.02 & 0.00 & 0.15 & 0.14 & 0.16 \\
\hline Total & 290.59 & 38.63 & 57.56 & 24.56 & 35.00 & \begin{tabular}{|l|}
17.93 \\
\end{tabular} & 16.91 \\
\hline
\end{tabular}

\section{CONCLUSION}

In this paper graph theory is applied to calculate the contribution factors of individual generators to line flows and loads and the extraction factors of individual loads from line flows and generators. The power transfer allocation between individual generators and loads are significant to transmission open access. Related lemmas have been proved to guarantee the feasibility of the method. Bus-inflow-line and bus-outflow-line incident matrices are built up to fast determine the downstream and upstream power flow tracing sequences. The DSTR is performed to determine the contribution factors of generations to the line flows and loads, whereas USTR is performed to determine the extraction factors of individual loads from line flows and generators. The suggested method is very efficient and suitable for use in real power systems. 


\section{APPENDIX}

TABLE II

LOAD FLOW OF THE IEEE 30-BUS SYSTEM

\begin{tabular}{l|c|c|c|c|c|c}
\hline Bus & \multicolumn{2}{|c|}{ Voltage } & \multicolumn{2}{c|}{ Generation } & \multicolumn{2}{c}{ Load } \\
\hline & $\begin{array}{c}\text { Amplitude } \\
\text { (p.u.) }\end{array}$ & $\begin{array}{c}\text { Angle } \\
\text { (degree) }\end{array}$ & $\begin{array}{c}\text { Active } \\
\text { (MW) }\end{array}$ & $\begin{array}{c}\text { Reactive } \\
\text { (MVAR) }\end{array}$ & $\begin{array}{c}\text { Active } \\
\text { (MW) }\end{array}$ & $\begin{array}{c}\text { Reactive } \\
\text { (MVAR) }\end{array}$ \\
\hline 1 & 1.05 & 0.00 & 138.63 & 3.13 & 0.00 & 0.00 \\
\hline 2 & 1.03 & -2.75 & 57.56 & 13.75 & 21.70 & 12.70 \\
\hline 3 & 1.03 & -4.59 & 0.00 & 0.00 & 2.40 & 1.20 \\
\hline 4 & 1.02 & -5.51 & 0.00 & 0.00 & 7.60 & 1.60 \\
\hline 5 & 1.01 & -9.04 & 24.56 & .26 .88 & 94.20 & 19.00 \\
\hline 6 & 1.02 & -6.43 & 0.00 & 0.00 & 0.00 & 0.00 \\
\hline 7 & 1.00 & -8.02 & 0.00 & 0.00 & 22.80 & 10.90 \\
\hline 8 & 1.02 & -6.52 & 35.00 & 48.89 & 30.00 & 30.00 \\
\hline 9 & 1.04 & -8.09 & 0.00 & 0.00 & 0.00 & 0.00 \\
\hline 10 & 1.03 & -10.02 & 0.00 & 0.00 & 5.80 & 2.00 \\
\hline 11 & 1.05 & -6.12 & 17.93 & 7.53 & 0.00 & 0.00 \\
\hline 12 & 1.03 & -9.09 & 0.00 & 0.00 & 11.20 & 7.50 \\
\hline 13 & 1.05 & -7.84 & 16.91 & 14.65 & 0.00 & 0.00 \\
\hline 14 & 1.02 & -10.04 & 0.00 & 0.00 & 6.20 & 1.60 \\
\hline 15 & 1.02 & -10.18 & 0.00 & 0.00 & 8.20 & 2.50 \\
\hline 16 & 1.02 & -9.78 & 0.00 & 0.00 & 3.50 & 1.80 \\
\hline 17 & 1.02 & -10.17 & 0.00 & 0.00 & 9.00 & 5.80 \\
\hline 18 & 1.01 & -10.85 & 0.00 & 0.00 & 3.20 & 0.90 \\
\hline 19 & 1.01 & -11.04 & 0.00 & 0.00 & 9.50 & 3.40 \\
\hline 20 & 1.01 & -10.85 & 0.00 & 0.00 & 2.20 & 0.70 \\
\hline 21 & 1.02 & -10.50 & 0.00 & 0.00 & 17.50 & 11.20 \\
\hline 22 & 1.02 & -10.49 & 0.00 & 0.00 & 0.00 & 0.00 \\
\hline 23 & 1.01 & -10.67 & 0.00 & 0.00 & 3.20 & 1.60 \\
\hline 24 & 1.01 & -10.96 & 0.00 & 0.00 & 8.70 & 6.70 \\
\hline 25 & 1.01 & -10.91 & 0.00 & 0.00 & 0.00 & 0.00 \\
\hline 26 & 1.00 & -11.33 & 0.00 & 0.00 & 3.50 & 2.30 \\
\hline 27 & 1.03 & -10.60 & 0.00 & 0.00 & 0.00 & 0.00 \\
\hline 28 & 1.01 & -6.85 & 0.00 & 0.00 & 0.00 & 0.00 \\
\hline 29 & 1.01 & -11.82 & 0.00 & 0.00 & 2.40 & 0.90 \\
\hline 30 & 1.00 & -12.70 & 0.00 & 0.00 & 10.60 & 1.90 \\
\hline Total & & & 290.59 & 114.81 & 286.40 & 126.20 \\
\hline & & & & & & \\
\hline
\end{tabular}

\section{REFERENCES}

[1] J. Bailey, "Tracing the flow of electricity," IEE Proceedings-Generation Transmission Distribution, vol. 143, no. 4, July 1996.

[2] J. Bialek, "Topological generation and load distribution factors for supplementary charge allocation in transmission open access," IEEE Trans. on PWRS, vol. 12, no. 3, Aug. 1997.

[3] D. Kirschen et al., "Contribution of individual generators to loads and flows," IEEE Trans. on PWRS, vol. 12, no. 1, Feb. 1997.

[4] K. Thulasiraman and M. Swamy, Graphs: Theory and Algorithms: John Wiley \& Sons, Inc., 1992.

[5] Y. Alavi et al., Graph Theory, Combinatorics, Algorithms and Applications: Society for Industrial and Applied Mathematics, 1991.

Felix F. Wu received his Ph.D. degree from University of California at Berkeley (UCB). He is now a Chair Professor of electrical engineering and Vice President of the University of Hong Kong. Prior to that he was a Professor and Vice-Chair of Department of EECS, UCB. His research interests are electric energy industry restructuring, power system investment planning, design of modern control centers, distribution automation, distributed processing etc.

Yixin Ni received her B.Eng., M.Eng., and Dr.Eng. degrees all in electrical engineering, Tsinghua University, China. She was former Professor and Director of National Power System Lab, Tsinghua University and now with the University of Hong Kong. Her research interests are power system stability and control, FACTS, AI tech. applications in power systems and power market.

Ping Wei received her M.Eng. degree in electrical engineering from Southeast University, China. She is now a Ph.D. student, Department of EEE, the University of Hong Kong. Her research area is power system operation, power market, and operations research applications in power systems. 